\title{
Treatment and outcomes of recurrent/persistent Cushing's disease: a single-center experience
}

\author{
Xiaohai Liu" ${ }^{1 \#}$, Congxin Dai ${ }^{2 \#}$, Xinjie Bao ${ }^{3}$, Kan Deng ${ }^{3}$, Yong Yao ${ }^{3}$, Bowen Sun ${ }^{2}$, Ming Feng $^{3}$, \\ Renzhi Wang ${ }^{3}$
}

${ }^{1}$ Department of Neurosurgery, Xuanwu Hospital Capital Medical University, Beijing, China; ${ }^{2}$ Department of Neurosurgery, Tongren Hospital Capital Medical University, Beijing, China; ${ }^{3}$ Department of Neurosurgery, Peking Union Medical College Hospital, Beijing, China

Contributions: (I) Conception and design: X Liu, R Wang; (II) Administrative support: M Feng, Y Yao; (III) Provision of study materials or patients: All authors; (IV) Collection and assembly of data: X Liu, C Dai; (V) Data analysis and interpretation: All authors; (VI) Manuscript writing: All authors; (VII) Final approval of manuscript: All authors.

\#These authors contributed equally to this work.

Correspondence to: Renzhi Wang. Department of Neurosurgery, Peking Union Medical College Hospital, Chinese Academy of Medical Sciences and Peking Union Medical College, Beijing 100730, China. Email: wangrz@126.com.

Background Although transsphenoidal adenomectomy is the first treatment choice for Cushing's disease (CD), tumor recurrence rates are as high as $45 \%$, resulting in a therapeutic challenge for endocrinologists and neurosurgeons. For recurrent/persistent CD (RCD/PCD), Repeat transsphenoidal surgery (RTSS), radiotherapy, gamma knife radiosurgery (GKRS) or bilateral adrenalectomy may be considered. The purpose of the study was to report the treatments and outcomes of RCD/PCD in a single center.

Methods: A retrospective study was performed on 55 RCD/PCD patients from a single center between 2000 and 2012 at Peking Union Medical College Hospital (PUMCH).

Results: Among the 55 RCD/PCD patients, 43 achieved remission (78\%), and 11 maintained a PCD status (20\%). The average times to recurrence after the initial treatment and Repeated treatment were $43.25 \pm 10.3$ and $5.13 \pm 4.7$ months, respectively $(\mathrm{P}=0.006) ; 17.6 \%$ of the patients experienced recurrence within the first year, and $52.9 \%$ of the patients experienced recurrence within 1.1-5.0 years. For the Repeated treatments, surgery was more effective for the biochemical remission of serum cortisol and adrenocorticotrophic hormone (ACTH) levels than radiotherapy. Delayed remission occurred in $28.9 \%(11 / 38)$ of the patients after Repeated surgery. Considering the previous biochemical levels after the initial surgery in RCD patients, $19.75 \%$ of patients experienced recurrence when serum cortisol levels were less than $<3 \mu \mathrm{g} / \mathrm{dL}$, and $51.4 \%$ of patients experienced recurrence under conditions of hypocortisolism and when 24-hour urinary free cortisol (24 UFC) was undetectable.

Conclusions: RTSS remains a recommended treatment option for RCD/PCD patients while radiotherapy is suggested as an adjunctive treatment. Intensive follow-up is recommended, as patients with serum cortisol levels $<3 \mu \mathrm{g} / \mathrm{dL}$ or undetectable 24 UFC levels can still experience recurrence after surgical treatment.

Koywords: Recurrent Cushing's disease (RCD); persistent Cushing's disease (PCD); repeated transsphenoidal surgery; radiotherapy; bilateral adrenalectomy.

Submitted Nov 16, 2020. Accepted for publication Nov 30, 2020.

doi: 10.21037/apm-20-2230

View this article at: http://dx.doi.org/10.21037/apm-20-2230 


\section{Introduction}

Cushing's disease (CD), caused by an adrenocorticotropic hormone (ACTH)-secreting pituitary adenoma, comprises $70 \%$ of endogenous Cushing's syndrome cases; Cushing's syndrome is associated with hypercortisolism and significant morbidity, especially metabolic and cardiovascular complications, osteoporosis, psychiatric changes, and cognitive impairment (1). The natural history of $\mathrm{CD}$ is associated with a $50 \% 5$-year survival rate (2). Although transsphenoidal adenomectomy is considered the first choice of treatment with immediate postoperative remission rates ranging between $65 \%$ and $90 \%$ (3), tumor recurrence rates are as high as $12-45 \%$, resulting in a great challenge for endocrinologist and neurosurgeons (3). Repeat transsphenoidal surgery (RTSS) is recommended as the treatment of choice for PCD/RCD after an initial transsphenoidal selective adenomectomy (4). Others do not favor re-exploration because of negative experiences $(5,6)$. However, no study to date has compared the efficacy of reoperation versus radiotherapy in PCD/RCD patients in a single center and identified the recurrencerelated clinical factors associated with RCD and PCD after repeat transsphenoidal surgery (RTSS) as a first-line treatment. This retrospective study reports the outcomes of transsphenoidal reoperation and radiotherapy in $55 \mathrm{PCD} /$ RCD patients at our institution.

We present the following article in accordance with the STROBE reporting checklist (available at http://dx.doi. org/10.21037/apm-20-2230).

\section{Methods}

\section{Patients}

A total of 530 patients were diagnosed CD and received TSS at Peking Union Medical College Hospital from 2000-2012, in which a total of 56 RCD/PCD patients were identified. $55 \mathrm{RCD} / \mathrm{PCD}$ patients were eligible for inclusion, and 1 patient was excluded due to the loss of medical records. Follow-up was conducted by phone calls and the examination of recent outpatient medical records. Historical medical records were also analyzed for patients who were lost to follow-up or died. All patients had at least 12 months of clinical follow-up data after their most recent treatment. Patients who were later diagnosed with Nelson's syndrome were also included. The medical reports (including surgical data) of patients who underwent their initial transsphenoidal surgery TSS at another institution were requested and reviewed. The study was approved by the Research Ethics Committee of Peking Union Medical College Hospital (S-K1465) and written informed consent was obtained from all the patients according to the institutional guidelines. The study conformed to the provisions of the Declaration of Helsinki (as revised in 2013).

\section{Diagnostic criteria for $R C D / P C D$}

RCD is defined as a reappearance of cushingoid symptoms and hypercortisolism after resection (1). Some studies required extended period (3-6 months) of eucortisolism to confirm remission, otherwise it was considered PCD (7), but there is not consensus on this definition. PCD is defined as a failure in complete resection with persistent cushingoid symptoms and hypercortisolism (8). As hypercortisolism may develop as a delayed and gradual cortisol decrease, a 3-month period is proposed for diagnostic confirmation (9). Our RCD patients were diagnosed according to the following criteria: (I) Patients experienced a relapse in cushingoid symptoms after confirmed remission or had PCD (remission not achieved). (II) Patients presented a positive pituitary mass on magnetic resonance imaging (MRI) and computed tomography (CT) imaging; the mass was evaluated for the laterality, location and size of the tumor. (III) Patients presented abnormal endocrine profiles, including a combination of abnormal serum cortisol levels (cortisol $>25 \mu \mathrm{g} / \mathrm{dL}$ ) and 24-hour urinary free cortisol (24 UFC) levels (24-hour urinary measurement for cortisol $>100.0 \mu \mathrm{g} / 24$ hour) and abnormal results of low-dose and high-dose dexamethasone suppression tests (LDDST, HDDST). (IV) Patients had complete histological data and immunostaining results after resection.

\section{Remission criteria}

Our remission criteria for RCD and $\mathrm{PCD}$ were as follows: (I) The 24 UFC level was reduced to eucortisolism levels (20-100 $\mathrm{\mu g} / 24$ hour), including hypocortisolism levels ( $<20 \mu \mathrm{g} / 24$ hour) and undetectable 24 UFC (10). (II) Serum cortisol levels decreased to within or below the normal range $(5.0-25.0 \mu \mathrm{g} / \mathrm{dL})$ and values lower than 5 or $3 \mu \mathrm{g} / \mathrm{dL}$ were specified $(10,11)$. Data were obtained after withdrawal of glucocorticoids. (III) The results of LDDSTs were normal during follow-up. (IV) Cushingoid symptoms were relieved, and no relapse recurred during follow-up. 
Table 1 Basic characteristics of patients underwent repeat treatments for recurrent Cushing's disease $(n=55)$

\begin{tabular}{|c|c|c|}
\hline Basic characteristics and data & Number & Range/\% \\
\hline Mean age (yr) & 35.1 & $8-58$ \\
\hline \multicolumn{3}{|l|}{ Gender } \\
\hline Male & 5 & $9 \%$ \\
\hline Female & 50 & $91 \%$ \\
\hline \multicolumn{3}{|l|}{ Outcome } \\
\hline Remission & 43 & $78 \%$ \\
\hline Recurrent until now & 11 & $20 \%$ \\
\hline \multicolumn{3}{|l|}{ Therapeutic choice after TSS $(n=50)$} \\
\hline Repeated surgical treatment only & 38 & $69 \%$ \\
\hline Radiotherapy after initial surgical treatment & 8 & $15 \%$ \\
\hline Radiosurgery after initial surgical treatment & 4 & $7 \%$ \\
\hline \multicolumn{3}{|l|}{ First surgical treatment $(n=52)$} \\
\hline Tumor presence under MRI & 40 & \\
\hline Immediate remission after surgery* & 44 & $83 \%$ \\
\hline Cavernous sinus invasion & 4 & \\
\hline Optic chiasm compression & 3 & \\
\hline \multicolumn{3}{|l|}{ Available immunological assays $(n=43)$} \\
\hline $\mathrm{ACTH}(+)$ & 41 & $95 \%$ \\
\hline ACTH(-) & 2 & $5 \%$ \\
\hline \multicolumn{3}{|l|}{ Second surgical treatment $(n=41)^{\$}$} \\
\hline Tumor presence under MRI & 39 & \\
\hline Immediate remission after surgery* & 27 & $66 \%$ \\
\hline Cavernous sinus invasion & 5 & \\
\hline Optic chiasm compression & 2 & \\
\hline \multicolumn{3}{|l|}{ Available immunological assays $(n=32)$} \\
\hline $\mathrm{ACTH}(+)$ & 29 & $91 \%$ \\
\hline $\mathrm{ACTH}(-)$ & 3 & $9 \%$ \\
\hline
\end{tabular}

*, immediate biochemical remission after surgery; ${ }^{\$}$, include patients performed radiotherapy or radiosurgery before second repetitive surgery.

\section{Treatment}

The treatment modalities depended upon the agreement of endocrinologists and neurosurgeons at PUMCH. Patients were informed of the risks and benefits for every clinical scenario and signed an informed consent form.
The treatment algorithm for RCD/PCD patients is shown below. TSS performed in our hospital by one surgical team (consisting of 9 members) under the guidance of senior neurosurgeon Renzhi Wang. Radiotherapy/gamma knife radiosurgery (GKRS) were our adjunctive options when the patient refused reoperation or there was a possible risk of mortality associated with the reoperation. The treatment option to choose radiotherapy or radiosurgery were decided by a multidisciplinary team (MDT) to which consisted of doctors with expertise in neurosurgery, endocrinology, radiotherapy, radiology, pathology and other specialties. Radiotherapy was performed by our hospital, and GKRS was performed by the Navy General Hospital in Beijing. Radiotherapy was performed using 3-dimensional conformal radiation therapy (3DCRT). The pituitary tumor region was exposed to $\mathrm{X}$-ray radiation (200 cGY) daily for a total of 25 days for an overall dose of 5,000 cGY over 5 weeks. Drug therapies, such as ketoconazole, were not used in this setting. If no evidence of a tumor was found under MRI-contrast imaging and there was no indication for surgical exploration, adrenalectomy was recommended by the consensus of our medical professionals.

\section{Statistical analysis}

Statistical analysis was performed with SPSS 15.0 software (SPSS, Inc., Chicago, IL, USA). Comparison of categorical variables was carried out by Chi-square or Fisher's exact tests. Binary logistic regression was employed to analyze independent predicted variables of clinical refractoriness. When two-sided $\mathrm{P}$ values were $\leq 0.05$, the differences were considered statistically significant.

\section{Results}

\section{General data}

The basic characteristics of the RCD/PCD patient data are summarized in Table 1. Of the initial 55 patients, 52 patients underwent TSS, 2 patients underwent radiosurgery and 1 patient underwent unilateral adrenalectomy (due to a misdiagnosis). Thirty-four patients (62\%) underwent initial TSS in our hospital, and 21 patients (38\%) underwent initial TSS in other qualified hospitals in China. The enrolled patients had their reoperations or treatments performed by professionals at our hospital.

Figure 1 shows the algorithm of 52 patients and each successive medical treatment. Fifteen patients experienced 


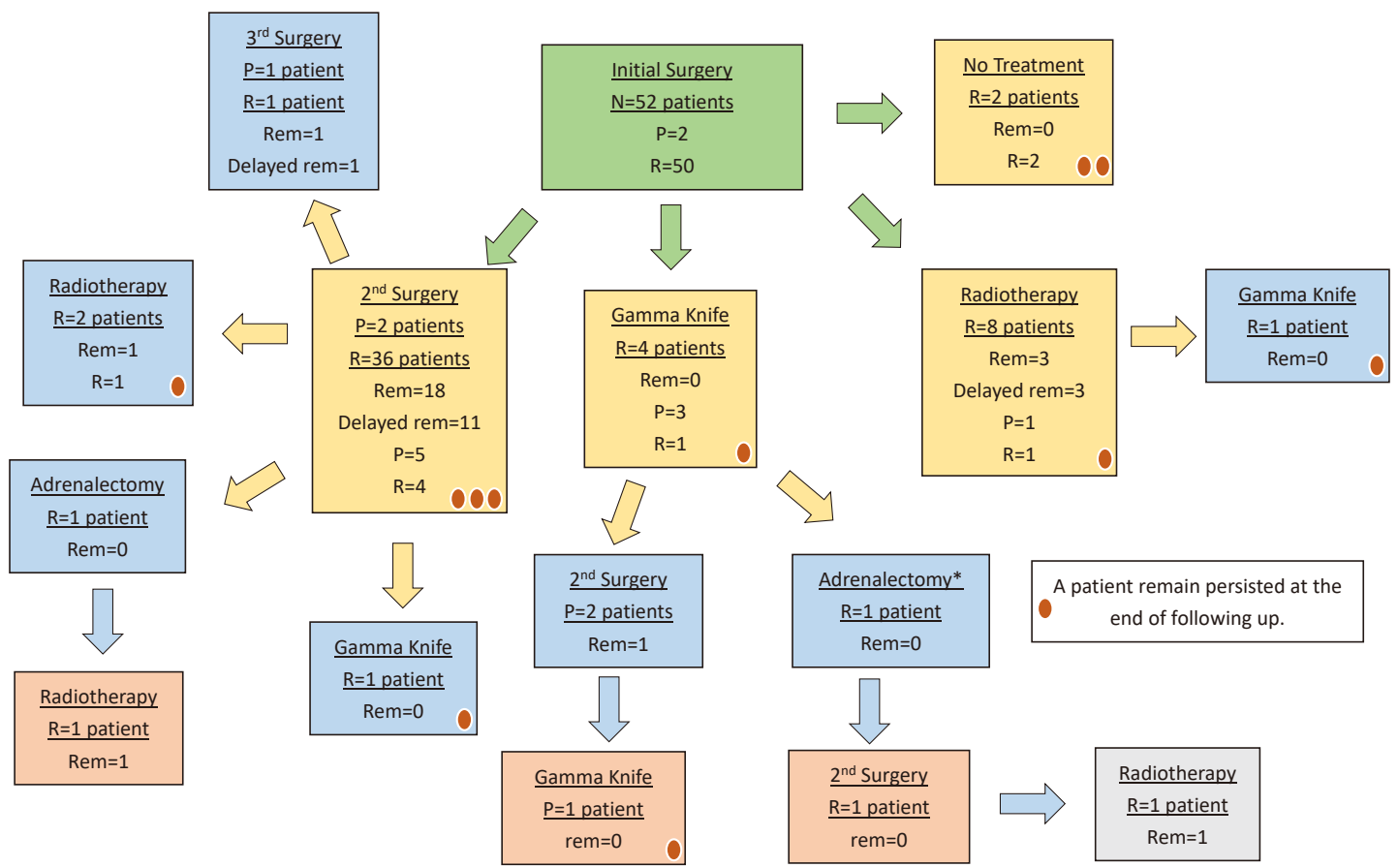

Figure 1 Treatment algorithm in 52 RCD patients (excluded 3 patients who initial treatment were not TSS). Each box presents each treatment scenario, the underlined $\mathrm{R}$ or $\mathrm{P}$ represent number of patients before treatment correlates whether patients were persistent or recurrent. The number that were not underlined represent number of patients after treatment. $\mathrm{P}=$ persistent, $\mathrm{r}=$ recurrence, rem $=$ remission, * = occur nelson's syndrome.

delayed remission, 12 patients experienced reoperation, and 3 patients experienced delayed remission after radiotherapy. At the end of the follow-up period, 43 patients (78\%) experienced complete remission, 11 patients $(20 \%)$ maintained a PCD status, and one patient (2\%) died (a forensic analysis was not performed). Three patients were diagnosed with Nelson's syndrome; 1 patient died without a forensic analysis, 1 patient maintained a PCD status, and 1 patient presented controlled biochemical levels and symptoms.

\section{Tumor size and pathological characteristics}

The tumor size and characteristics were derived from the available MRI/CT and surgical reports. Among the 55 patients, only 53 radiological reports were available. Forty-one patients $(77.35 \%)$ were diagnosed with microadenoma $(<10 \mathrm{~mm})$, with a mean size of $4.66 \pm 0.31 \mathrm{~mm}$, and 12 patients $(22.64 \%)$ were diagnosed with macroadenomas, with a mean size of $20.25 \pm 6.17 \mathrm{~mm}$. There were 6 patients who were initially diagnosed with macroadenomas after recurrence, and 9 patients were diagnosed with initial macroadenomas that recurred as microadenomas. Cavernous sinus invasion, optic chiasm compression and protrusion of the suprasellar level all occurred in 2 patients who had tumor masses larger than $40 \mathrm{~mm}$, and the disturbances were resolved after resection. For the second round of treatment, the radiological data of 51 patients were available; 43 patients were diagnosed with microadenomas (mean size $7.37 \pm 0.77 \mathrm{~mm}$ ) and 8 patients were diagnosed with macroadenomas (mean size $23.10 \pm 11.77 \mathrm{~mm}$ ). Once the tumor was resected, it was transferred immediately to a neuropathologist for histological confirmation and an immunoassay for diagnostic differentiation (see Table 1).

\section{Time to recurrence}

For the 55 patients, we calculated the time span of RCD, starting from the patient's approximated first onset of cushingoid symptoms to the final outcome of recurrence or resolution. Table 2 shows the time required for patients to receive treatment and the time of postoperative recurrence; the data were derived from the medical records and followup information of patients. Of the 51 RCD patients who 
Table 2 Time required for patients to seek successive treatments and tumor's recurrence after treatment (including TS, radiotherapy or radiosurgery)

\begin{tabular}{|c|c|c|c|c|}
\hline Time set & \multicolumn{2}{|c|}{ Number of patients } & \multicolumn{2}{|c|}{ Time (months) } \\
\hline Initial onset $\rightarrow$ first treatment & 55 & 0 & $31.2 \pm 8.8$ & $1-169$ \\
\hline First treatment $\rightarrow$ first $R C D / P C D$ & 55 & 0 & $43.25 \pm 10.3$ & $0-173$ \\
\hline First treatment $\rightarrow$ RCD (exclude PCD) & 51 & - & $46.02 \pm 10.3$ & $2-173$ \\
\hline Second treatment $\rightarrow$ second RCD/PCD & 18 & 35 & $5.13 \pm 4.7$ & $0-26$ \\
\hline Second treatment $\rightarrow$ RCD (exclude PCD) & 9 & - & $8.80 \pm 6.3$ & $1-26$ \\
\hline Second RCD/PCD $\rightarrow$ third treatment & 13 & $5^{\#}$ & $19.5 \pm 10.7$ & $1-65$ \\
\hline Third treatment $\rightarrow$ third RCD/PCD & 8 & 5 & $3.25 \pm 2.6$ & $1-7$ \\
\hline
\end{tabular}

${ }^{\$}$, discharge can be either resolution or refuse for further treatment; *, includes persistent after medical treatment; ${ }^{*}$, refuse further treatment.

underwent initial treatment (excluding 4 PCD patients), 9 patients (17.6\%) experienced recurrence within the first year after surgery, 27 patients $(52.9 \%)$ experienced recurrence within 1.1-5.0 years after surgery, and 14 patients $(27.4 \%)$ experienced recurrence more than 5.0 years after surgery. As shown in Table 2, after each successive treatment, the tumor had a tendency to recur quicker, and the first, second and third tumor recurrence occurred $43.25 \pm 10.3,5.13 \pm 4.7$, and $3.25 \pm 2.6$ months after the previous tumor, respectively. The time between the first treatment and second treatment had a $\mathrm{P}$ value of 0.006 , but the time between the second treatment and third treatment had a $\mathrm{P}$ value of 0.603 , showing no clinical significance. In order to analyze the relationship between cortisol level and tumor recurrence, we separated the RCD patients into 3 groups according to their previous serum cortisol level after initial surgery: a $<3 \mu \mathrm{g} / \mathrm{dL}$ group, a $3-8 \mu \mathrm{g} / \mathrm{dL}$ group and a $>8 \mu \mathrm{g} / \mathrm{dL}$ group. The remission rates were $19.75 \%$, $25 \%$ and $55.25 \%$, respectively. Moreover, after initial surgery, the time required for tumor recurrence between the serum cortisol $\mathrm{F}<2 \mu \mathrm{g} / \mathrm{dL}$ group and the cortisol $>2 \mu \mathrm{g} / \mathrm{dL}$ group was not significantly different; the average time to recurrence in 6 patients (cortisol $<2 \mu \mathrm{g} / \mathrm{dL}$ ) was $76.77 \pm 55.67$ months (range 16 to 159 ), and the average time in the rest of the patients was $43.25 \pm 10.44$ months. The $\mathrm{P}$ value was 0.107 .

\section{Endocrine data after surgical treatment and radiotberapy}

Most postoperative endocrinologic assessments were conducted within a week; unfortunately, not all the enrolled patients had complete serum cortisol, ACTH or 24 UFC data. Considering the available data, Table 3 shows the changes in the serum cortisol ( $8 \mathrm{am})$, ACTH and 24 UFC levels before and after treatments. The LDDSTs and HDDSTs were performed to identify and differentiate the types of CD. Before the initial TSS, CD in $86.7 \%$ of patients could not be suppressed by low-dose dexamethasone, and CD in $28.6 \%$ of patients could not be suppressed by high-dose dexamethasone. Before the second treatment, CD in $91.1 \%$ of patients was suppressed by lowdose dexamethasone, and CD in $10.3 \%$ of patients was suppressed by high-dose dexamethasone. Regarding the profiles of the 55 RCD patients, 6 patients reached serum cortisol levels $<2 \mu \mathrm{g} / \mathrm{dL}$ after the initial surgical treatment, and 3 patients reached these levels after Repeated treatment. No patients had cortisol levels lower than $<2 \mu \mathrm{g} / \mathrm{dL}$ after radiosurgery or radiotherapy.

There were 16 patients (out of 31 patients with available data) who reached hypocortisolism and undetectable 24 UFC levels after the initial TSS and 4 patients (out of 36 patients with available data) who reached these levels after reoperation. Hypocortisolism and undetectable 24 UFC levels were not observed in the radiotherapy/radiosurgery group. Of the 55 RCD patients who underwent initial TSS, $74.1 \%$ of the microadenoma patients achieved biochemical remission, with $40 \%$ of the patients presenting undetectable 24 UFC levels or serum cortisol levels $<2 \mu \mathrm{g} / \mathrm{dL}$. For the macroadenoma patients, $77.78 \%$ reached biochemical remission to the normal range, with $42.5 \%$ presenting undetectable 24 UFC levels or 
Table 3 Biochemical changes before and after medical treatments

\begin{tabular}{|c|c|c|c|c|}
\hline Variable & $\mathrm{n}$ & Before treatment $^{+}$ & After treatment $^{+}$ & $P$ value \\
\hline \multicolumn{5}{|l|}{ Serum cortisol ( $\mu \mathrm{g} / \mathrm{dL})$} \\
\hline Overall 1st treatment ${ }^{\#}$ & 37 & $26.96 \pm 3.41$ & $15.65 \pm 7.66$ & 0.000 \\
\hline Overall 2nd treatment ${ }^{\#}$ & 47 & $26.06 \pm 2.32$ & $20.04 \pm 5.12$ & 0.045 \\
\hline 1st surgical treatment ${ }^{\$}$ & 25 & $26.99 \pm 4.45$ & $17.46 \pm 10.10$ & 0.002 \\
\hline Radiotherapy* & 7 & $25.94 \pm 8.69$ & $24.38 \pm 17.83$ & 0.189 \\
\hline \multicolumn{5}{|l|}{ ACTH $(p g / m L)^{+}$} \\
\hline Overall 1st treatment ${ }^{\#}$ & 38 & $117.59 \pm 65.9$ & $42.15 \pm 21.63$ & 0.01 \\
\hline Overall 2nd treatment ${ }^{\#}$ & 50 & $72.54 \pm 11.46$ & $35.24 \pm 8.21$ & 0.000 \\
\hline Radiotherapy* & 7 & $64.34 \pm 33.95$ & $28.26 \pm 19.87$ & 0.175 \\
\hline \multicolumn{5}{|l|}{24 UFC ( $\mu \mathrm{g} / 24 \mathrm{hr})$} \\
\hline Overall 1st treatment ${ }^{\#}$ & 31 & $338.57 \pm 114.2$ & $141.52 \pm 129.1$ & 0.078 \\
\hline Overall 2nd treatment ${ }^{\#}$ & 48 & $518.26 \pm 140.47$ & $585.79 \pm 205.50$ & 0.740 \\
\hline 1st surgical treatment ${ }^{\$}$ & 23 & $372.71 \pm 151.51$ & $79.94 \pm 61.89$ & 0.001 \\
\hline 2nd surgical treatment ${ }^{\$}$ & 37 & $532.76 \pm 176.71$ & $662.91 \pm 101.12$ & 0.986 \\
\hline Radiotherapy* & 6 & $511.54 \pm 195.75$ & $241.14 \pm 350.35$ & 0.160 \\
\hline
\end{tabular}

${ }^{+}$, ACTH data for Nelson's syndrome were excluded; $"$, includes surgery, radiotherapy and radiosurgery; ${ }^{\$}$, only performed initial and repeated surgical treatment; ${ }^{*}$, radiotherapy performed in 2 nd treatment.

serum cortisol levels $<2 \mu \mathrm{g} / \mathrm{dL}$.

\section{Complications after reoperation}

Nineteen patients with cerebrospinal fluid (CSF) leakage due to TSS recovered; 18 patients underwent operative skull base reconstruction, and 1 patient required a Repeated operation. Two macroadenoma patients required extensive respiratory support due to hospital-acquired pneumonia, but they recovered. One patient experienced cardiac arrest due to hyperkalemia 2 weeks postoperatively and was successfully resuscitated. Postoperative panhypopituitarism occurred in 6 patients. None of the patients experienced meningitis or vision loss during treatment.

\section{Unilateral/bilateral adrenalectomy}

When patients experienced a relapse in CD symptoms but were negative on MRI and had minimal success with
Repeated TSS, unilateral/bilateral adrenalectomy was performed. Of the 55 total patients, 4 patients underwent adrenalectomy, 1 patient underwent unilateral adrenalectomy, and 3 patients underwent bilateral adrenalectomy. One of the patients underwent adrenalectomy as the initial treatment. Regarding the final outcomes, 3 patients achieved remission, and one patient died of an unknown cause. Three patients were diagnosed with Nelson's syndrome after adrenalectomy; regarding their final outcomes, 1 patient died of an unknown cause, 1 patient maintained a PCD status, and 1 patient had controlled biochemical levels and symptoms.

\section{Radiosurgery}

Eight patients underwent GKRS from 1998 to 2011. As shown in Table 4, none of them achieved complete remission. Six patients underwent GKRS after initial TSS, 2 patients required Repeated TSS to achieve remission, 1 patient required both bilateral adrenalectomy and TSS to 
Table 4 Treatments and outcomes of patients who performed Gamma knife surgery in combination with other treatments

\begin{tabular}{|c|c|c|c|c|c|}
\hline Case\# & 1st treatment & 2nd treatment & 3rd treatment & 4th treatment & Outcome \\
\hline 2 & TSS & $\mathrm{R}$ & GKR & - & Recurrence \\
\hline 3 & TSS & TSS & GKR & - & Recurrence \\
\hline 4 & TSS & GKR & TSS & - & Remission \\
\hline 6 & TSS & GKR & B & $\mathrm{R}$ & Remission \\
\hline 7 & GKR & TSS & B & GKR & Death* \\
\hline 8 & GKR & TSS & TSS & - & Remission \\
\hline
\end{tabular}

TSS, transsphenoidal surgery; GKR, Gamma knife radiation; R, radiotherapy; B, bilateral adrenalectomy; *, unknown cause.

achieve remission, and 3 patients experienced recurrence and refused further treatment. Thus, the remission rate was $42.8 \%$. There were 2 patients who underwent GKRS before surgery, 1 patient who achieved remission after Repeated TSSs, and another patient who was diagnosed with Nelson's syndrome and died due to unknown causes.

\section{Discussion}

From 2000 to 2012, 475 CD patients received TSS and achieved complete remission; the remission rate was $89.6 \%$. Fifty-five RCD/PCD patients received further treatments, and the overall remission rate increased to $97.7 \%$; 43 patients achieving remission and 12 patients maintained a PCD status. In a previous study, the majority of CD patients were female patients aged 35-41 years old (12). This figure is similar to that in our study; the average age of the $530 \mathrm{CD}$ patients was 34.1 years and in the 55 RCD/PCD patients was 35.1 years. Among the $530 \mathrm{CD}$ patients, 101 patients were male and 429 were female, with a male to female ratio of $1: 4.3$, similar to the global ratio of 1:5 (1). However, whether sex influences recurrence in Asian populations is unclear.

Although all patients were recommended to undergo secondary clinical treatment, these 55 patients chose different tailored treatment modalities depending upon affordability and their attitude towards reoperation. Most importantly, there was no evidence or consensus to suggest that repetitive surgical intervention was more effective than radiotherapy or radiosurgery in the Asian population. Remission criteria are weighted differently and biochemical remission varies depending upon the clinical treatment selection. After surgical intervention, professionals considered normal or subnormal cortisol levels ( $<50 \mathrm{nmol} / \mathrm{L} 5-14$ days postoperatively) necessary to declare remission (13). These levels are associated with a low recurrence rate of approximately $10 \%$ in 10 years. Other researchers believe that the normalization of UFC is enough to declare remission (14). For radiosurgery or radiotherapy, the remission standard focuses on the relief of cushingoid symptoms and the normalization of UFC levels without the use of medications (15). Immediate remission is rarely achievable once a normal UFC is reached, and long-term remission can be expected. Chen $e t$ al. showed that serum cortisol levels of 3-8 $\mu \mathrm{g} / \mathrm{dL}$ should be closely monitored, and medical staff should be required to inform patients of the poor prognosis (16). Trainer also found that no patients with a postoperative serum cortisol level of $50 \mathrm{nmol} / \mathrm{L}(1.8 \mathrm{~g} / \mathrm{dL})$ or less experienced recurrence (17). Here, we separated the RCD patients into 3 groups according to their previous serum cortisol level after initial surgery: a $<3 \mu \mathrm{g} / \mathrm{dL}$ group, a $3-8 \mu \mathrm{g} / \mathrm{dL}$ group and a $>8 \mu \mathrm{g} / \mathrm{dL}$ group. The remission rates were $19.75 \%$, $25 \%$ and $55.25 \%$, respectively; thus, we cannot simply stop monitoring when the patient meets the remission standard of $<3 \mu \mathrm{g} / \mathrm{dL}$. Interestingly, those with serum cortisol levels $>8 \mu \mathrm{g} / \mathrm{dL}$ accounted for the majority of RCD patients. Atkinson suggested that subnormal serum cortisol levels should not be the only marker of remission, as the majority of patients, even those within the normal range, can still achieve long-term remission (18).

A consensus statement suggests that long-term remission can be expected when decreased 24 UFC levels are detected, whereas levels within a normal range of $20-100 \mu \mathrm{g}$ is equivocal; values above the normal range indicate a persistent tumor (19). One study noted that 
undetected cortisol levels also cannot effectively predict outcomes (20), similar to our results. According to the previous 24 UFC after initial surgery in RCD patients, hypocortisolism and undetected 24 UFC levels were present in $51.4 \%$ of the group; $29.03 \%$ had levels within the normal range, and $19.35 \%$ had levels above the normal range. Thus, predicting the prognosis considering a confirmed biochemical cutoff point may not be reliable. Recurrence is possible under any postoperative condition; thus, regular monitoring for recurrence remains necessary (21). Most CD patients can achieve immediate remission after initial surgery, but most RCD patients require an extended period to achieve remission.

The time to recurrence varied among the RCD patients after each successive treatment. On the basis of our results, a long-term follow-up period of up to 4-6 years is crucial to conclude whether CD patients are more likely to be free from recurrence after resection. A meta-analysis showed that RCD patients will experience the highest frequency of recurrence between 1-5 years, in comparison with recurrence at $<1$ or $>5$ years (22). In addition, our results also showed that $17.6 \%$ of the patients experienced recurrence within the first year, and $52.9 \%$ experienced recurrence within 1.1-5.0 years. Thus, an ideal timescale for follow-up after initial treatment is 5 years, but lifelong monitoring remains crucial.

On average, RCD patients experienced a second onset of CD symptoms 45.8 months after their initial surgery. Patients required an average of 31.2 months for medical treatment (either surgical treatment or radiosurgery), with 169 months being the longest time from the first onset of symptoms. A lack of medical attention and a lack of access to a general practitioner or adequate facilities and professionals may be the reason for the extended treatment time. Table 2 shows that after each successive treatment, the tumor had a tendency to recur quicker, and the first, second and third tumor recurrence occurred $43.25 \pm 10.3,5.13 \pm 4.7$, and $3.25 \pm 2.6$ months after the previous tumor, respectively. The time between the first treatment and second treatment had a $\mathrm{P}$ value of 0.006 , but the time between the second treatment and third treatment had a $\mathrm{P}$ value of 0.603 , showing no clinical significance. This gradient was due to factors including increased difficulty in Repeated resection or possibly aggressive behavioral changes after stimulation from the surgical intervention, radiotherapy and radiosurgery.

Surgical skills and experience are important factors in achieving remission, and many studies have concluded that inexperienced surgeons often failed to perform a complete resection, unlike experienced professionals (23). Unlike most studies in which TSS was performed by a surgeon to prevent inherent variability, our current experience suggests that junior surgeons guided by senior professionals can also obtain a remission rate of $97.7 \%$. Careful intraoperative bilateral exploration with repetitive rinsing with saline remains effective for microadenoma if the tumor was soft. Normally, recurrent or residual tumors are located immediately contiguous to the original tumor site (24)

Of the 38 patients who underwent reoperation after the initial surgery (no intervention with radiotherapy or radiosurgery, Figure 1), 18 patients achieved immediate remission, and 11 patients reached delayed remission, with a remission rate of $76.3 \%$. The remission rate after reoperation is $43-61 \%$ (4). A total of $28.9 \%(11 / 38)$ of the RCD patients presented delayed remission after reoperation, but this is rarely reported in studies. Valassi showed delayed remission after initial surgery in $5.6 \%$ of the patients who presented early elevated or normal UFC levels. These patients presented a delayed and persistent cortisol decrease after an average of $38 \pm 50$ postoperative days (9).

A second TSS is generally more difficult to perform than the initial operation (25). The existing scar tissue, irregular anatomical landmarks, and aftermath of radiotherapy/ radiosurgery may contribute to the difficulty encountered in Repeated TSS; complications are often inevitable (18). However, common complications can be prevented with careful management (24). Thirty-four percent of our RCD patients had CSF leakage during the operation; $94.7 \%$ of the patients underwent operative skull base reconstruction, and one patient underwent Repeated repair surgeries. None of our patients experienced meningitis. One patient experienced cardiovascular arrest one week after the operation, but resuscitation was successful. Other studies also noted similar complications and concluded that lethal postoperative cardiovascular events may occur within 2 weeks (26).

Radiotherapy along with ketoconazole is generally used as an adjuvant therapy following surgical resection, especially in patients with residual tumors who are poorly suited for reoperation (27). The outcome can be either an immediate resolution or several years of delayed remission (18-24 months on average). However, in China, drug therapy is not generally used along with radiotherapy. Table 3 shows that conventional radiotherapy is less effective than surgical treatment, but it remains a promising 
adjunctive treatment for patients who refuse further invasive operations. Radiotherapy is useful against any type of endocrine-secreting pituitary adenoma can produce a biochemical remission rate of $56-83 \%$ in CD patients and is indicated for persistent hypercortisolism after surgery (28). In our study, 6 of 8 patients (75\%) achieved biochemical remission with radiotherapy; 3 patients achieved immediate remission and the other 3 patients experienced delayed remission. None of the patients experienced radiotherapeutic-related complications, such as optic nerve damage.

GKRS is considered a regular treatment for RCD in European countries when Repeated TSS fails (29). Studies have shown that the therapeutic effects outweigh the effects of radiotherapy; GKRS is associated with a reliable antiproliferative effect, but it may require an average of 22 months to reach biochemical remission (30). Many studies documented that GKRS after initial TSS decreased the tumor size by $54-80 \%$, and $42.5-78 \%$ of patients achieved biochemical remission within 5 years (normalization of 24 UFC levels). A total of $7-20 \%$ of patients in remission may experience a relapse (19-60 months posttreatment), but Repeated GKRS can achieve biochemical remission in $42-76 \%$ of RCD patients (29). Unfortunately, none of our patients achieved remission with GKRS alone (12 to 60 months of observation), and they required extensive treatment, such as Repeated surgeries and radiotherapy to achieve remission, with a remission rate of $42.8 \%$. As shown in Table 4, when RCD patients underwent GKRS as their final medical treatment, they tended to have poor outcomes. Thus, radiosurgery as an adjuvant therapy may not be suitable for the Asian population, although complications such as visual deficits or cranial nerve deficits were not observed (31). Table 3 shows that 24 UFC levels were not different before and after surgical intervention, with a $\mathrm{P}$ value of 0.986 ; thus, if we could obtain 24 UFC levels at the time when the patient reached remission, we could evaluate the importance of UFC levels in the decision for reoperation.

\section{Conclusions}

At present, the long-term follow-up of patients who underwent Repeated TSS confirms this as an effective treatment for PCD/RCD, and a combination of TSS and radiotherapy remains necessary for RCD. Our medical intervention proved to be effective for the Asian population, and delayed remission is common after the second TSS. A minimum of 5 years of follow-up is recommended. Reduced postoperative endocrine levels including serum cortisol levels $<3 \mu \mathrm{g} / \mathrm{dL}$ or undetectable 24 UFC levels are not completely reliable, and close monitoring is recommended.

\section{Acknowledgments}

Funding: The financial support for this study was provided by the Scientific Research Project of Capital Health Development in 2018 (grant number: 2018-4-4018) and the Beijing Natural Science Foundation (grant number: 7182137). The funding institutions had no role in the design of the study, data collection and analysis, decision to publish, or preparation of the manuscript.

\section{Footnote}

Reporting Checklist: The authors have completed the STROBE reporting checklist. Available at http://dx.doi. org/10.21037/apm-20-2230

Data Sharing Statement: Available at http://dx.doi. org/10.21037/apm-20-2230

Conflicts of Interest: All authors have completed the ICMJE uniform disclosure form (available at http://dx.doi. org/10.21037/apm-20-2230). The authors have no conflicts of interest to declare.

Etbical Statement: The authors are accountable for all aspects of the work in ensuring that questions related to the accuracy or integrity of any part of the work are appropriately investigated and resolved. The study was approved by the Research Ethics Committee of Peking Union Medical College Hospital (S-K1465) and written informed consent was obtained from all the patients according to the institutional guidelines. The study conformed to the provisions of the Declaration of Helsinki (as revised in 2013).

Open Access Statement: This is an Open Access article distributed in accordance with the Creative Commons Attribution-NonCommercial-NoDerivs 4.0 International License (CC BY-NC-ND 4.0), which permits the noncommercial replication and distribution of the article with the strict proviso that no changes or edits are made and the original work is properly cited (including links to both the formal publication through the relevant DOI and the license). See: https://creativecommons.org/licenses/by-nc-nd/4.0/. 


\section{References}

1. Nishioka H, Yamada S. Cushing disease. J Clin Med 2019;8:1951.

2. Hammer GD, Tyrrell JB, Lamborn KR, et al. Transsphenoidal microsurgery for Cushing's disease: initial outcome and long-term results. J Clin Endocrinol Metab 2004;89:6348-57.

3. Lacroix A, Feelders RA, Stratakis CA, et al. Cushing's syndrome. Lancet 2015;386:913-27.

4. Rubinstein G, Osswald A, Zopp S, et al. Therapeutic options after surgical failure in Cushing's disease: A critical review. Best Pract Res Clin Endocrinol Metab 2019;33:101270.

5. Jagannathan J, Smith R, DeVroom HL, et al. Outcome of using the histological pseudocapsule as a surgical capsule in Cushing disease. J Neurosurg 2009;111:531-9.

6. Jagannathan J, Sheehan JP, Pouratian N, et al. Gamma Knife surgery for Cushing's disease. J Neurosurg 2007;106:980-7.

7. Patil CG, Veeravagu A, Prevedello DM, et al. Outcomes after repeat transsphenoidal surgery for recurrent Cushing's disease. Neurosurgery 2008;63:266-70.

8. Bertagna X, Guignat L. Approach to the Cushing's disease patient with persistent/recurrent hypercortisolism after pituitary surgery. J Clin Endocrinol Metab 2013;98:1307-18.

9. Valassi E, Biller BM, Swearingen B, et al. Delayed remission after transsphenoidal surgery in patients with Cushing's disease. J Clin Endocrinol Metab 2010;95:601-10.

10. Braun LT, Rubinstein G, Zopp S, et al. Recurrence after pituitary surgery in adult Cushing's disease: a systematic review on diagnosis and treatment. Endocrine 2020;70:218-31.

11. Santoro A, Minniti G, Ruggeri A, et al. Biochemical remission and recurrence rate of secreting pituitary adenomas after transsphenoidal adenomectomy: longterm endocrinologic follow-up results. Surg Neurol 2007;68:513-8.

12. Dekkers OM, Biermasz NR, Pereira AM, et al. Mortality in patients treated for Cushing's disease is increased, compared with patients treated for nonfunctioning pituitary macroadenoma. J Clin Endocrinol Metab 2007;92:976-81.

13. Rutkowski MJ, Flanigan PM, Aghi MK. Update on the management of recurrent Cushing's disease. Neurosurg Focus 2015;38:E16.

14. Kim JH, Shin CS, Paek SH, et al. Recurrence of Cushing's disease after primary transsphenoidal surgery in a university hospital in Korea. Endocr J 2012;59:881-8.

15. Castinetti F, Brue T, Ragnarsson O. Radiotherapy as a tool for the treatment of Cushing's disease. Eur J Endocrinol 2019;180:D9-18.

16. Chen JC, Amar AP, Choi S, et al. Transsphenoidal microsurgical treatment of Cushing disease: postoperative assessment of surgical efficacy by application of an overnight low-dose dexamethasone suppression test. J Neurosurg 2003;98:967-73.

17. Trainer PJ, Lawrie HS, Verhelst J, et al. Transsphenoidal resection in Cushing's disease: undetectable serum cortisol as the definition of successful treatment. Clin Endocrinol (Oxf) 1993;38:73-8.

18. Atkinson AB, Kennedy A, Wiggam MI, et al. Long-term remission rates after pituitary surgery for Cushing's disease: the need for long-term surveillance. Clin Endocrinol (Oxf) 2005;63:549-59.

19. Biller BM, Grossman AB, Stewart PM, et al. Treatment of adrenocorticotropin-dependent Cushing's syndrome: a consensus statement. J Clin Endocrinol Metab 2008;93:2454-62.

20. Yap LB, Turner HE, Adams CB, et al. Undetectable postoperative cortisol does not always predict long-term remission in Cushing's disease: a single centre audit. Clin Endocrinol (Oxf) 2002;56:25-31.

21. Santoro A, Minniti G, Ruggeri A, et al. Biochemical remission and recurrence rate of secreting pituitary adenomas after transsphenoidal adenomectomy: longterm endocrinologic follow-up results. Surg Neurol 2007;68:513-8.

22. Roelfsema F, Biermasz NR, Pereira AM. Clinical factors involved in the recurrence of pituitary adenomas after surgical remission: a structured review and meta-analysis. Pituitary 2012;15:71-83.

23. Shikary T, Andaluz N, Meinzen-Derr J, et al. Operative Learning Curve After Transition to Endoscopic Transsphenoidal Pituitary Surgery. World Neurosurg 2017;102:608-12.

24. Hofmann BM, Hlavac M, Kreutzer J, et al. Surgical treatment of recurrent Cushing's disease. Neurosurgery 2006;58:1108-18.

25. Bunevicius A, Laws ER, Vance ML, et al. Surgical and radiosurgical treatment strategies for Cushing's disease. J Neurooncol 2019;145:403-13.

26. Burke WT, Penn DL, Repetti CS, et al. Outcomes After Repeat Transsphenoidal Surgery for Recurrent Cushing Disease: Updated. Neurosurgery 2019;85:E1030-6.

27. Capatina C, Hinojosa-Amaya JM, et al. Management of 
patients with persistent or recurrent Cushing's disease after initial pituitary surgery. Expert Rev Endocrinol Metab 2020;15:321-39.

28. Hughes JD, Young WF, Chang AY, et al. Radiosurgical Management of Patients With Persistent or Recurrent Cushing Disease After Prior Transsphenoidal Surgery: A Management Algorithm Based on a 25-Year Experience. Neurosurgery 2020;86:557-64.

29. Bunevicius A, Sheehan D, Lee Vance M, et al. Outcomes of Cushing's disease following Gamma Knife radiosurgery: effect of a center's growing experience and era of treatment. J Neurosurg 2020;31:1-8.

30. Castinetti F, Nagai M, Dufour H, et al. Gamma knife radiosurgery is a successful adjunctive treatment in Cushing's disease. Eur J Endocrinol 2007;156:91-8.

31. Sheehan JP, Xu Z, Salvetti DJ, et al. Results of Gamma Knife surgery for Cushing's disease. J Neurosurg 2013;119:1486-92.

Cite this article as: Liu X, Dai C, Bao X, Deng K, Yao Y, Sun B, Feng M, Wang R. Treatment and outcomes of recurrent/ persistent Cushing's disease: a single-center experience. Ann Palliat Med 2021;10(3):2494-2504. doi: 10.21037/apm-20-2230 